\title{
Técnica de uso do corpo adiposo da bochecha para reconstrução orbitária
}

Technique of use of the cheek fat body for orbital reconstruction

Técnica de uso del cuerpo grasiento de la mejilla para la reconstrucción orbital

Breno dos Reis FERNANDES
Darah Lígia MARCHIORI $^{\mathbf{1}}$
Oswaldo BELLOTI NETO $^{\mathbf{3}}$
Patrese Pereira de BELLA $^{\mathbf{3}}$
Gabriel MULINARI-SANTOS $^{\mathbf{1}}$
Patrick Peloso Pereira FIGUEIREDO
Fernando Luiz Chiella BUCHELE $^{\mathbf{3}}$
Jonathan Ribeiro da SILVA
Antonio Marcos dos SANTOS

1 Faculdade de Odontologia de Araçatuba, UNESP-Univ. Estadual Paulista, Araçatuba-SP, 16015-050 Araçatuba-SP, Brasil

${ }^{2}$ Universidade Alto Vale do Rio do Peixe, UNIARP - Campus Caçador, 89500-000 Caçador-SC, Brasil

${ }^{3}$ Hospital das Clínicas de Teresópolis Constantino Ottaviano HCTCO, 25976-016 Teresópolis-RJ, Brasil

\section{Resumo}

O corpo adiposo da bochecha é uma massa tubular de gordura localizado no espaço bucal, entre os músculos masseter e o bucinador, auxiliando nos processos de sucção e mastigação, além de desempenhar uma função protetora de algumas estruturas da face. Devido à sua rica vascularização, tamanho semelhante em todos os indivíduos, fácil acesso e pequena taxa de complicações, é usado para vários fins terapêuticos. Neste artigo é relatado um caso clínico no qual o corpo adiposo de bichat é utilizado em uma reconstrução orbitária para dar volume ao conteúdo orbitário.

Descritores: Tecido Adiposo; Corpo Adiposo; Cirurgia Bucal.

\section{Abstract}

The cheek adipose body is a tubular mass of fat located in the oral space, between the masseter muscles and the buccinator, assisting in the sucking and chewing processes, in addition to playing a protective role in some face structures. Due to its rich vascularity, similar size in all individuals, easy access and low rate of complications, it is used for various therapeutic purposes. In this article a clinical case is reported in which the bichat adipose body is used in an orbital reconstruction to add volume to the orbital content.

Descriptors: Adipose Tissue; Fat Body; Surgery, Oral.

\section{Resumen}

El cuerpo adiposo de la mejilla es una masa tubular de grasa ubicada en el espacio oral, entre los músculos maseteros y el buccinador, que ayuda en los procesos de succión y masticación, además de desempeñar un papel protector en algunas estructuras de la cara. Debido a su rica vascularización, tamaño similar en todos los individuos, fácil acceso y baja tasa de complicaciones, se utiliza para diversos fines terapéuticos. En este artículo se informa un caso clínico en el que el cuerpo adiposo bichat se utiliza en una reconstrucción orbital para agregar volumen al contenido orbital.

Descriptores: Tejido Adiposo; Cuerpo Adiposo; Cirugía Bucal.

\section{INTRODUÇÃO}

O corpo adiposo da bochecha se localiza na bochecha, entre os músculos masseter e bucinador, que tem como função proteger estruturas neuromusculares da face, além de auxiliar no processo de sucção e mastigação. $\mathrm{O}$ corpo adiposo da bochecha anatomicamente possui o corpo e quatro prolongamentos, com um rico suprimento sanguíneo ${ }^{1}$.

Devido sua nutrição e fácil acesso, essa gordura é de interesse cirúrgico, podendo ser utilizada como um enxerto livre ou pediculado para fechar defeitos maxilares após a excisão de tumores, no encerramento de comunicação oroantral pósextração, fístula oroantral e fissura palatina ${ }^{2,3}$.

O tamanho do corpo adiposo da bochecha é constante entre pessoas e não depende do peso corporal total e/ou distribuição de gordura, pela facilidade de acesso e rico suprimento sanguíneo, o corpo adiposo da bochecha é adequeado para reconstruções de defeitos orais ${ }^{4}$.

Devido à sua localização, existem alguns riscos cirúrgicos associados, tais como os relacionados com a lesão de estruturas nobres, levando a parestesia, paralisia e assimetria facial, além de dificuldade de abertura da boca, e possibilidade de uma posterior flacidez ${ }^{2}$.

\section{CASO CLÍNICO}

Paciente do sexo masculino, 21 anos, leucodema, vítima de acidente motociclístico, foi encaminhado ao pronto socorro do Hospital das Clinicas de Teresópolis/RJ. Ao exame clínico inicial apresentava ferida cortocontusa em região de pálpebra superior e queixava-se de dor em região orbitária direita, diplopia e enoftalmia (Figura 1).

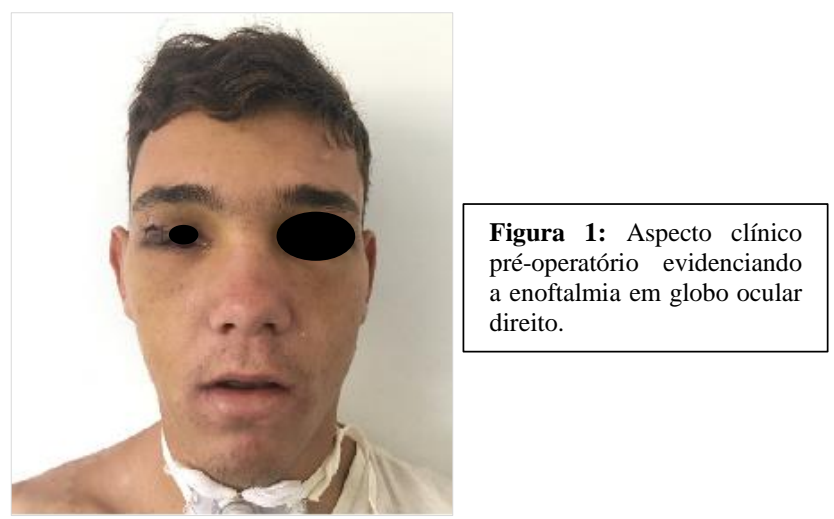

Após exame clinico inicial foi solicitado tomografia de face, no qual era possível constatar fratura de rebordo infraorbital direito, assoalho orbital direito além de fratura da parede lateral de orbita direita e fratura de pilar zigomático maxilar direito, além de lipólise de conteúdo orbital diante do 
quadro clinico do paciente o mesmo foi internado pela especialidade para posterior reconstrução do complexo orbitário. Após 3 dias o paciente foi encaminhado para o centro cirúrgico para realização da cirurgia reconstrutiva de orbita direita, sob anestesia geral e intubação nasotraqueal. Foi realizado um único acesso subciliar com cantotomia, para chegar nas áreas fraturadas em região orbitaria além de um acesso vestibular maxilar para acessar a região de pilar zigomático. (Figuras 2, 3, 4 e 5).

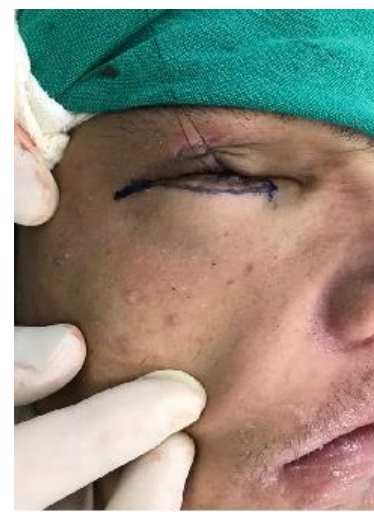

Figura 2: Marcação do acesso subciliar com cantotomia.

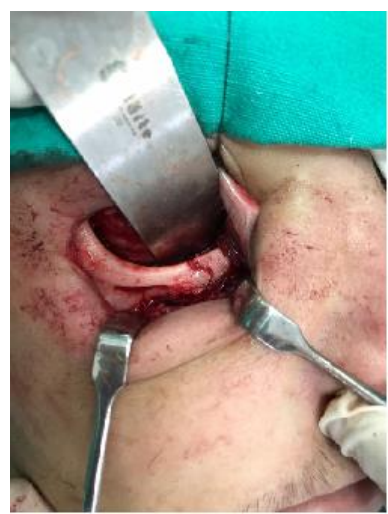

Figura 3: Exposição do rebordo infraorbital assoalho orbital direito.

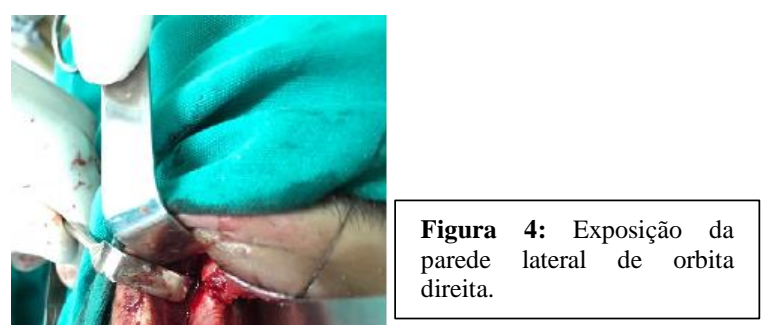

Figura 5: Acesso Caldwell Luc, utilizado para expor a fratura de pilar zigomático maxilar, além da exposição do corpo adiposo de Bichat.
Logo em seguida realizou-se a redução da região zigomática além da fixação do pilar zigomático-maxilar utilizando uma placa em L do sistema de 2.0mm (Figura 6).

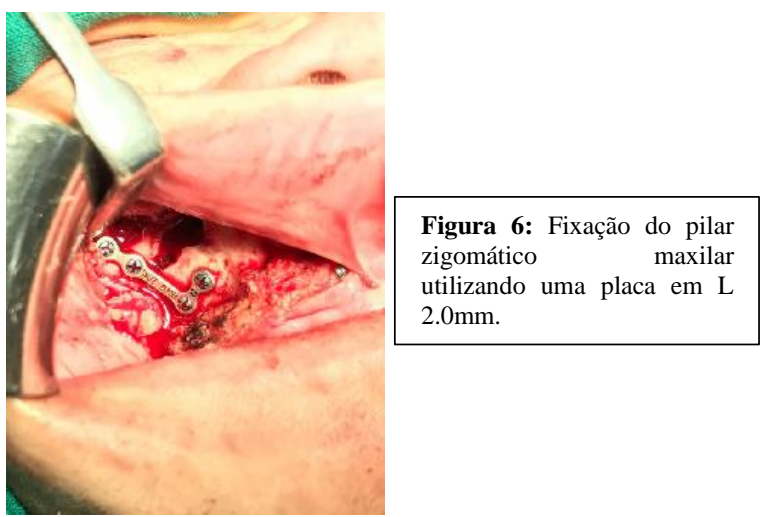

Posteriormente foram realizadas redução e fixação da parede lateral de orbita direita, utilizando uma placa de 4 furos de 1,5mm (Figura 7), e a reconstrução do rebordo infraorbital com uma placa orbitaria de 6 furos do sistema $1,5 \mathrm{~mm}$ e uma malha de titânio foi utilizada para reconstruir o assoalho orbital (Figura 8).

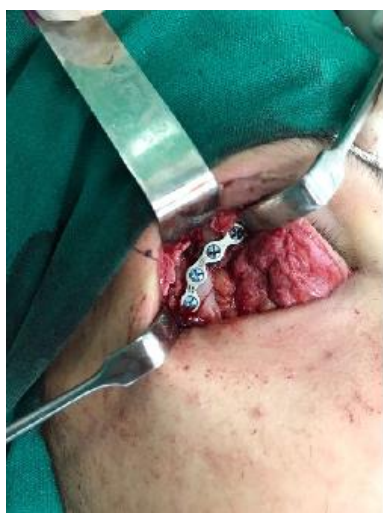

Figura 7: Fixação da parede lateral de orbita utilizando uma placa de 4 furos do sistema de $2.0 \mathrm{~mm}$.

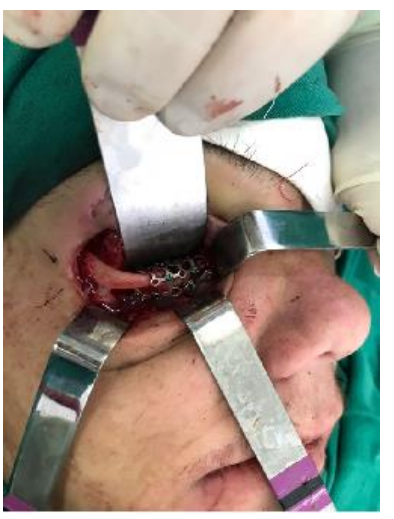

Figura 8: Fixação do
rebordo infra orbital
utilizando uma placa orbital
de 6 furos do sistema $1.5 \mathrm{~mm}$,
além da reconstrução do
assoalho orbital utilizando
uma malha de titânio.

Como o paciente havia perdido parte da gordura orbital, aproveitando o acesso vestibular maxilar realizado intraoral, foi removido parte do corpo adiposo da bochecha, para auxiliar no ganho de conteúdo orbitário (Figura 9). Após a reconstrução os tecidos profundos foram suturados com Vycril $3.0 \mathrm{e} \mathrm{a}$ pele com Nylon 5.0. Após 02 dia de pós-operatório o paciente havia apresentado melhoras significativa na enoftalmia além da ausência de diplopia recebendo alta pela especialidade para acompanhamento 
ambulatorial, com prescrição de: ibuprofeno 400mg e dipirona 500mg a cada 6 horas por 03 dias, além de amoxicilina 500mg a cada 8 horas por 07 dias. No sétimo dia de pós-operatório foi realizado a remoção de sutura, e mantido acompanhamento ambulatorial semanal por 8 semanas, mensal por 3 meses, e após apenas semestral. Hoje o paciente apresenta 06 messes de pós-operatório sem intercorrências. (Figura 10).
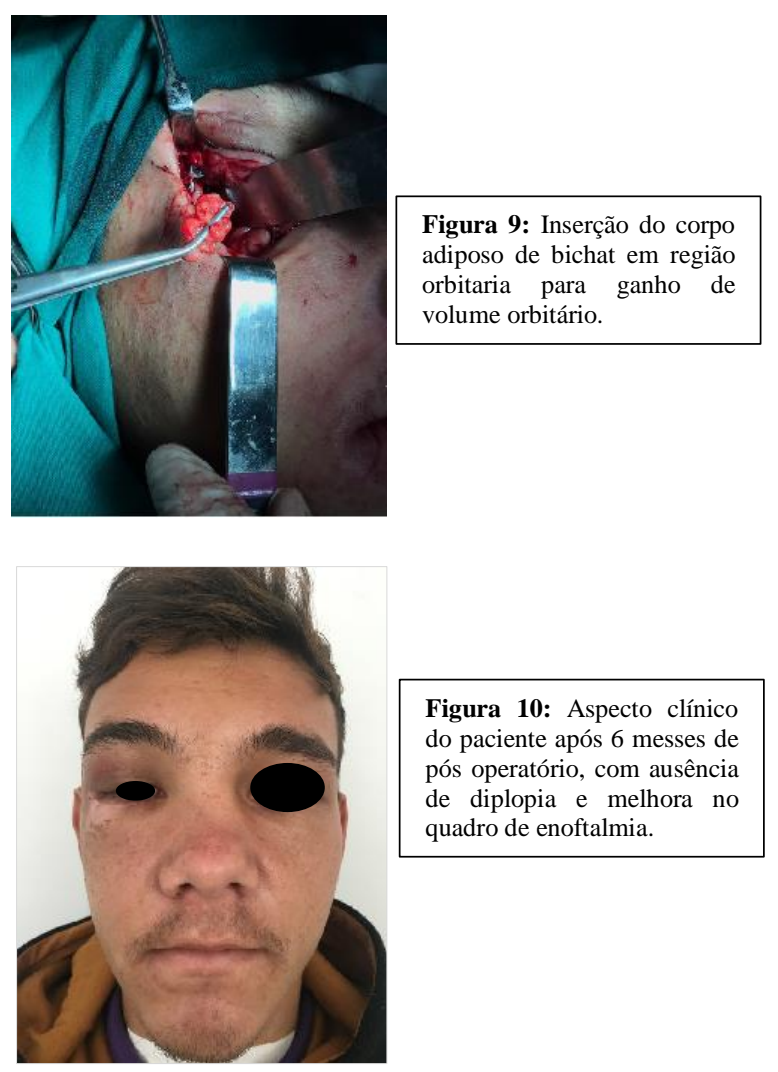

DISCUSSÃO

O corpo adiposo da bochecha foi descrito por Marie-Francois Xavier Bichat, anatomista Francês de forte relevância em sua época, em 1802, baseado em seus estudos de autopsia introduziu inúmeras estruturas que levaram seu nome, como a fossa pterigopalatina, chamada inicialmente de fossa de Bichat e a protuberância de Bichat, popularmente conhecido como bola de Bichat, que se refere ao corpo adiposo bucal ${ }^{5}$. O qual está localizado no denominado espaço mastigatório, e apresenta peso médio de 9,3 gramas, e o volume em média de $9,6 \mathrm{ml}$, com pouca variação entre o lado direito e esquerdo, em torno de 1,5 gramas, sendo normalmente removido uma quantidade de 4 a 6 gramas em ambos os lados ${ }^{6,7}$. O corpo adiposo da bochecha trata-se de uma gordura branca, pura, com poucas trabéculas fibrosas, sendo diferente da gordura subcutânea por ser muito mais fibrosa, talvez por esse fato ter sido descrita primeiramente por Heister, em 1732, como uma glândula, o mesmo apresenta um corpo principal e quatro extensões: bucal, pterigoideal, temporal superficial e profunda ${ }^{6}$. A partir das características de encapsulamento, ligamentos e nutrição por artérias, o corpo adiposo bucal pode ser divido em 3 lóbulos, anterior, intermediário e posterior. E as extensões, bucal, pterigoidea, pterigopalatina, e temporal são extensões derivadas do lóbulo posterior do corpo adiposo bucal ${ }^{5}$. A extensão bucal do lóbulo posterior do corpo adiposo bucal, é a porção responsável pelo contorno facial, confere plenitude à bochecha, corresponde a parte inferior do lóbulo posterior abaixo do ducto parotídeo, sendo a extensão mais superficial, assim seu volume pode afetar a aparência facial, e existe, de forma estável, ao longo de toda a vida do indivíduo. Já as extensões, temporal profunda, superior do corpo da gordura e a extensão pterigoidea são acessíveis acima do arco zigomático e parecem ter pequena influência no contorno facial ${ }^{8}$.

Apesar da intervenção cirúrgica do corpo adiposo da bochecha para adequação de contorno facial, devido ao fato desta estrutura anatômica conferir plenitude à bochecha e ser responsável pelo contorno facial, ter se tornado popular nos últimos anos, inúmeras outras aplicações clínicas do uso do corpo adiposo bucal na odontologia estão elencadas na literatura, entre elas: adequação do contorno facial nos casos de hipertrofia dos masseteres, reparação de defeitos por ressecções tumorais, por cistos maxilares, por comunicação oroantral, para correção de defeitos pós-traumáticos, reconstrução de defeitos do palato duro e do palato mole, utilizada como material preenchedor estético para dar volume em lábios, pré-maxila, paranasal; na região malar, preenchedor nos casos de perfuração da membrana do seio maxilar ${ }^{9-12}$.

\section{CONSIDERAÇÕES FINAIS}

Conclui-se que, o corpo adiposo da bochecha tem uma utilização versátil na cirurgia oral e maxilo facial em termos de localização e aplicação, podendo ser usado sozinho ou em combinação com outras técnicas, para o encerramento de defeitos ósseos e mucosos, e o sucesso desta técnica é atribuído à rica vascularização, menor morbidade no local doador, tamanho médio constante em todos os indivíduos, segurança e facilidade de colheita, além de menor taxa de complicações.

\section{REFERÊNCIAS}

1. Shoja MM, Tubbs RS, Loukas M, Shokouhi G, Ardalan MR. Marie-François Xavier Bichat (1771-1802) and his contributions to the foundations of pathological anatomy and modern medicine. Ann Anat.2008;190(5):413-20

2. Alonso-González $\mathrm{R}$, Peñarrocha-Diago $\mathrm{M}$, Peñarrocha-Oltra D, Aloy-Prósper A, CamachoAlonso F, Peñarrocha-Diago M. Closure of oroantral communications with Bichat's buccal fat pad. Level of patient satisfaction. J Clin Exp Dent. 2015;7(1):e28-33.

3. Peñarrocha-Oltra D, Alonso-González R, PellicerChover H, Aloy-Prósper A, Peñarrocha-Diago M. 
Closure of oroantral communication with buccal fat pad after removing bilateral failed zygomatic implants: A case report and 6-month follow-up. J Clin Exp Dent. 2015;7(1):e159-62.

4. Baumann A, Ewers R. Application of the buccal fat pad in oral reconstruction. J Oral Maxillofac Surg. 2000;58(4):389-92.

5. Zhang HM, Yan YP, Qi KM, Wang JQ, Liu ZF. Anatomical structure of the buccal fat pad and its clinical adaptations. Plast Reconstr Surg. 2002; 109(7):2519-20.

6. Stuzin JM, Wagstrom L, Kawamoto HK, Baker TJ, Wolfe SA. The anatomy and clinical application of the buccal fat pad. Plast Reconstr Surg. 1990;85(1):29-37.

7. Pessa JE, Rohrich RJ. Discussion: aging changes of the midfacial fat compartments: a computed tomographic study. Plast Reconstr Surg. 2012; 129(1):274-75.

8. Xu J, Yu Y. A modified surgical method of lowerface recontouring. Aesth Plast Surg. 2013; 37(2):216-21.

9. Martin-Granizo R, Naval L, Costas A, Goizueta $\mathrm{C}$, Rodriguez F, Monje $\mathrm{F}$ et al. Use of buccal fat pad to repair intraoral defects: review of 30 cases. Br J Oral Maxillofac Surg. 1997;35(2):81-4.

10.Carbonell A, Salavert A, Planas J. Resection of the Buccal Fat Pad in the Treatment of Hypertrophy of the Masseter Muscle. Aesthetic Plast Surg. 1991;15(3):219-22

11. Meyer E, Liebenberg SJ, Fagan JJ. Buccal fat pad-a simple underutilised flap. S Afr J Surg. 2012; 50(2): 47-9.

12.Berrone M, Florindi FU, Carbone V, Aldiano C, Pentenero M. Stage 3 medication-related osteonecrosis of the posterior maxilla: surgical treatment using a pedicled buccal fat pad flap: case reports. J Oral Maxillofac Surg. 2015; 73(11): 2082-86.

\section{CONFLITO DE INTERESSES}

Os autores declaram não haver conflitos de interesse.

\section{AUTOR PARA CORRESPONDENCIA}

\section{Gabriel Mulinari dos Santos}

gabriel_mulinari@hotmail.com

Submetido em 12/07/2019

Aceito em 21/09/2019 